\title{
CYCLING CHARITY CHALLENGE EVENTS: CAN THEY CONTRIBUTE TO THE LIFESTYLE MEDICINE MOVEMENT?
}

\author{
ALEXANDRA COGHLAN \\ Department of Tourism, Leisure, Hotel and Sport Management, Griffith University, \\ Gold Coast Campus Southport, QLD, Australia
}

\begin{abstract}
Many developed countries, including Australia, are experiencing an increase in lifestyle-related disease such as obesity, heart disease, and mental distress. A new model of health care known as lifestyle medicine encourages individuals to be active partners in their health care and take responsibility for their long-term health, as well as address causes of lifestyle-related disease and concentrate on prevention. Focusing on a cycling-based charity challenge event designed to raise funds to support cardio health, the Yellow Pages Cardiac Challenge, this article considers the role of charity challenge events, and more broadly cycle tourism, within lifestyle medicine. Using survey-based primary and secondary data, the study addresses two questions: To what extent do the socio-demographic profiles of charity challenge participants and lifestyle disease "at-risk" groups overlap and what is the cycling involvement and experience of charity challenge participants? The results suggest that charity challenge events can play a role in introducing individuals to the physical health benefits of cycling, and encourage mental health and well-being through the creation of new social relationships, development of a sense of achievement, and experiencing positive emotions. Whereas a number of questions remain to be answered, this study's results contribute to existing health policy debates by exploring the role of sports tourism in supporting lifestyle medicine initiatives.
\end{abstract}

Key words: Charity challenge events; Lifestyle movements; Health; Lifestyle medicine

\section{Introduction}

Australia, as well as other developed nations, faces an increasing health burden from major lifestylerelated diseases. Chronic, noninfectious diseases such as obesity, heart disease, cancer, diabetes, mental disorders, and musculoskeletal diseases account for a large proportion of the disease burden in this country (Egger \& Egger, 2012). Lifestyle medicine represents one new model of health care designed to manage lifestyle-related diseases and focus on changes in lifestyle patterns and behaviors. In this article, I consider how cycling tourism may contribute to lifestyle medicine through the relatively new subsector known as charity challenges. Charity 
challenges are participatory events that combine touring activities with extended physical activity, social, environmental, or health awareness campaigns, and fundraising for charity. As charity challenges include overnight stays away from home, and generally travel more than $40 \mathrm{~km}$, they may be considered forms of ("slow") tourism, using the definition adopted in Australia's National Visitors' Survey (Tourism Research Australia, 2013).

Linking charity fundraising with cycling events appears to particularly popular, not least within Australia (e.g., Brisbane's Ride for Life, South Australia's Ride to Cure Diabetes, Victoria's Ride4Epilepsy). As an active, mobile form of tourism transport, cycling can effectively link tourism with physical activity, active lifestyles, and sport participation. When this type of event participation or attendance includes overnight travel away from home, Lamont (2009a) suggests that it be included in a definition of cycling tourism in Australia as "participation in cycling may include attendance at events organized for commercial gain and/or charity (competitive and non-competitive)" (p. 20). Cycling charity challenge events may support lifestyle medicine initiatives by encouraging individuals to be active partners in care, take responsibility for their long-term health, address medial and proximal causes of lifestyle-related disease, and focus on primary, secondary, and tertiary prevention. By focusing on one particular event, a cycling charity challenge, this article explores how charity challenges can play a role in lifestyle movements and contribute to better health outcomes for participants. Adopting both a conceptual approach and providing some descriptive, quantitative data, the research explores overlaps between lifestyle disease "at-risk" group characteristics, cycling charity challenge participant characteristics, and participants' involvement with cycling, their motivations to participate in the event, and their cycling-related behaviors.

\section{Literature Review}

\section{Charity Challenges as a Form} of Lifestyle Movement

As a form of travel, charity challenges represent a relatively new subsector within the general area of alternative, slow tourism. They offer participants "the experience of travel, the experience of a life-time, with fundraising, travel and challenge combined with an altruistic purpose" (Goodwin, McCombes, \& Eckardt, 2009, p. 66). In most cases, the charity promotes a prescheduled event, involving overnight travel away from the usual place of residence, chooses the level of challenge and thrill, and/or may specifically tie the challenge to the charitable purpose. Challenge activities typically include hiking, cycling, kayaking, or a combination of activities. Cycling charity challenges appear to be particularly popular, perhaps because cycling is easily accessible, requires little specialist equipment (as a recreational activity), and represents a form of mobility suited to slow tourism. Cycling charity challenges may be competitive (e.g., the Mark Webber Challenge), community based (e.g., the Yellow Pages Cardiac Challenge), or more travel oriented (e.g., InspiredAdventures). Beneficiaries include health-based charities (e.g., the Cancer Foundation, Leukemia Foundation), conservation charities (e.g., Greenpeace), animal welfare charities (e.g., RSPCA), and human rights charities (e.g., Amnesty International), among others.

Details of the size and scope of charity challenge events are lacking; it is difficult to estimate the number of people involved in such events. However, anecdotal evidence suggests that this is a growing event sector, with new events established each year and an increasing number of participants registering for existing events. An online search using the term “charity challenge" results in 115,000,000 results, including a number of large-scale, commercial providers (e.g., i-to-i volunteering, World Expeditions), national and international not-forprofit organizations (e.g., Inspired Adventures), and smaller community events (e.g., the Yellow Pages Cardiac Challenge). Furthermore, Everyday Hero, a website designed to facilitate awareness of charity challenge events and fundraising for community events, states that it has helped more than 359, 000 participants raise over AU\$100 million (Everyday Hero, 2012).

As well as representing a form of alternative travel, charity challenges may be conceptualized as a form of lifestyle movement (i.e., a new form of social movement). Lifestyle movements are characterized as individualized collective action that 
consciously and actively promotes a lifestyle or way of life as their primary means to foster social change (Haenfler, Johnson, \& Jones, 2012). These types of movements stand in contrast to traditional social movements, which are politically oriented and carried out by structurally located collectives (e.g., based around gender, class, ethnicity) and, in contrast, subcultures, which do not focus on change, but instead seek to create a cultural space to freely express themselves. Instead, lifestyle movements coalesce around "communities of meaning" (Cohen, 1985), are value oriented, individualized in action, and aimed at fostering change. Examples include the green movement, the slow movement, the social responsibility movement, and the simple life (or voluntary simplicity) movement.

Being less structured than traditional social movements, lifestyle movements often provide repertoires of actions that set up alternative lifestyle and examples for promoting conscious shifts in behaviors in everyday life. Furthermore, Haenfler et al. (2012) argue that lifestyle movements generally focus on both potential external and personal benefits. Indeed, some individuals may choose a lifestyle primarily for their own health, mental, or financial benefits, thereby seeking personal change, and are less concerned with the societal changes that arise from their individual actions. At other times, it is concern for the environment, human equity, sociocultural well-being, or animal welfare more broadly that drives changes in behaviors and involvement in a lifestyle movement (e.g., Roubanis, 2008).

In the case of cycling charity challenges, it is hypothesized that charity challenges provide both personal and external benefits that are attractive to participants. At a fundamental level, Lyons and Wearing (2008) argue that charity challenges provide an attractive alternative to traditional travel by combining the experience of travel with a unique life time experience while raising funds for charity, thereby "blending the voluntary act of fund-raising with the more hedonic pleasures of a packaged adventure tour” (p. 151). The external benefits of charity challenges most likely include raising funds to enable not-for-profit organizations to do their charitable work, raising awareness of the charitable cause, as well as associated benefits such as low carbon travel through the use of nonmotorized transport, and increased social capital through the relationships developed prior to and during the event.

The more personal benefits of charity challenges could include opportunities to connect with the self and others (Coghlan \& Filo, 2013), enduring involvement in the activity (McIntyre, 1989), health benefits from the physical activity (in this case, cycling), and a sense of personal empowerment as a result of realizing fundraising goals. Other personal benefits may include enhanced personal well-being; charity challenges are frequently designed in such a way that foster social and emotional well-being, thriving, or flourishing. This social and emotional well-being effect may occur in many ways prior to, during, and after the event. For example, thriving is encouraged by combining stressors, adversity, vulnerabilities (the "challenge") with individual and social resources, the activation and development of strengths, a sense of agency and meaning, while promoting health and fitness outcomes (Massey, Cameron, Ouellette, \& Fine, 1998). Flourishing, on the other hand, may be fostered through intentional activity, motivation, and goal attainment, all of which represent pathways to well-being, activation of signature strengths, positive emotions and gratification, and sharing positive events (Frederickson, 2001; Gable, Reis, Impett, \& Asher, 2004; Lyubomirsky, Sheldon, \& Schkade, 2005; Seligman, Parks, \& Steen, 2004; Seligman, Steen, Park, \& Peterson, 2005; Vallerand, 2007). Many of these personal benefits are tied to Deci and Ryan's (1985) selfdetermination theory, which highlights the importance of competence, autonomy, and relatedness in fostering health and well-being in people, particularly where participation is intrinsically motivated.

\section{Charity Challenges, Health, and Lifestyle Medicine}

Given the potential personal benefits of charity challenges described above, it is proposed that such events can play a major role in combating lifestyle-related diseases, raising awareness of health issues, and improving preventative health measures. Cardiovascular disease and obesity are considered national health priorities within Australia as they affect one in seven and three in five 
Australians, respectively (Australian Institute of Health and Welfare [AIWH], 2012). More broadly, chronic, noninfectious diseases such as obesity, heart disease, cancer, diabetes, mental disorders, and musculoskeletal diseases account for a large proportion of the disease burden in Australia, with hospital admissions increasing by 37\% over the last decade, and GP visits also on the rise (Egger \& Egger, 2012).

Obesity is one health issue that is of major concern in Australia, and is linked to additional health concerns such as a cardiovascular disease, high blood pressure, and type 2 diabetes. The financial costs to society are also significant; research by the Australian Bureau of Statistics (ABS, 2011) noted that in 2008 the cost to Australia was AU\$58.2 billion, including a burden of disease cost of AU\$49.9 billion (cost of disability, loss of well-being, and premature death), and a direct financial cost of AU\$8.3 billion (employment impacts, health system costs, and carer costs). The increasing issue of obesity is directly linked to sedentary lifestyles created by increasing affluence and modernization of society and changes in diets towards more energy-dense foods. The ABS survey also provides obesity profiles (notwithstanding genetic predisposition), segmenting respondents by demographic and socioeconomic characteristics (including gender, age, employment, income, education, location) and physical activity. These profiles are provided in Table 1, and will be used to compare the profile of obesity sufferers with the profile of charity challenge participants.
Cardiovascular disease, including coronary heart disease, stroke, heart failure, rheumatic heart disease, and peripheral vascular disease is another major health concern for Australia, affecting about 3.5 million Australians in 2007-2008, and responsible for more deaths than any other disease group. According to the most recent figures released by the Australian Institute of Health and Welfare (AIWH, 2011), cardiovascular disease accounted for about $18 \%$ of the overall burden of disease in Australia in 2003. A number of risk factors have been identified for cardiovascular disease. A number of these, such as age, gender, ethnicity, and family history of the disease, cannot be changed. However, cardiovascular disease is included as a lifestyle disease, as many risk factors such as smoking, lack of exercise, being overweight, excessive alcohol use, and a poor diet are all related to lifestyle and behaviors and can therefore be changed to reduce the likelihood of cardiovascular disease. Furthermore, psychosocial factors, such as depression and social isolation, can also affect the development of cardiovascular disease.

Psychological distress (stress and lowered wellbeing) represent another significant health issue for Australians, and one that is commonly interlinked with obesity and cardiovascular disease. Mental health was declared a National Health Priority area in 1996 because of its impact on the Australian community; according to the Australian Psychological Society's annual Stress and Wellbeing survey ( $n=1,552$ ), up to $12 \%$ of respondents experience stress in the severe ranges (APS, 2012a). A further

Table 1

The Relationship Between Obesity, Cardiovascular Disease, and Psychological Distress With Key Sociodemographic Variables Within the Australian Community

\begin{tabular}{llll}
\hline & \multicolumn{1}{c}{ Obesity $^{\mathrm{a}}$} & Cardiovascular Disease $^{\mathrm{b}}$ & \multicolumn{1}{c}{ Psychological Distress $^{\mathrm{c}}$} \\
\hline Gender & Male & Male and females & Females \\
Age & Middle to late adulthood & Middle to late adulthood & Young adults \\
Education & Year 11 completed & Data not available & Lower education levels \\
Occupation & F/T employment, machinery & Data not available & Managerial and professionals \\
& operators, managers & Lowest income & Lowest income \\
Income & Lowest income & Regional and remote & Regional and remote \\
Location & Regional and remote & Exercise 2 days or less & Exercise 2 days or less \\
Physical activity & Exercise 2 days or less & & \\
\hline
\end{tabular}


33\% of Australians reported experiencing depressive symptoms with $10 \%$ of these being in the severe range. Young adults (18 to 25 age group) appear particularly vulnerable, and reported experiencing significantly higher levels of stress and significantly lower levels of well-being than the general population, as well as significantly higher levels of anxiety and depression. Women were significantly more likely to report higher levels of perceived stress than men, particularly with regards to personal health issues.

The literature presented here suggests that charity challenges can be usefully conceptualized as a form of lifestyle movement, allowing people to express their values on certain issues related to the charitable beneficiary, as well derive personal benefits from participation. Charity challenges also represent a form of alternative, slow tourism, as they involve overnight travel away from participants' usual place of residence, and cycling, as a form of soft mobility, appears to be a particularly popular activity for charity challenges. Participation in cycling as a form of soft mobility also bridges the areas of tourism and sport participation and may encourage health benefits for participants, as they not only support the values surrounding the behavior but also begin to integrate it into their lifestyles (Deci \& Ryan, 2000; Lamont \& Kennelly, 2012). Given the increase of lifestyle-related diseases and the new focus on lifestyle medicine, charity challenges may have a role to play in combating lifestyle-related disease, with important implications for public health policy. The question remains, however, whether the charity challenge participants display similar profiles to lifestyle disease "at-risk" groups, and can be encouraged to adopt new physically active lifestyles, or whether this approach is "preaching to the converted."

\section{Aims and Methods}

This research investigates the potential role of cycling charity challenges in improving health outcomes with particular reference to the major lifestyle-related diseases of concern in Australia (obesity, cardiovascular disease, and mental distress). The research is exploratory, and focuses primarily on profiling respondents in a cycling event to determine the potential of charity challenges to target lifestyle-related disease "at-risk" groups. The research is particularly concerned with the following two questions:

1. To what extent do charity challenge participant sociodemographic profiles overlap with "atrisk" group characteristics provided in Table 1 ?

2. What is the cycling involvement and experience of charity challenge participants?

To address these questions, a review of lifestylerelated diseases was undertaken (see above) and the characteristics of typical "at-risk" groups were identified based on common sociodemographic characteristics and physical activity participation rates. Next, a survey of charity challenge participants was undertaken with the assistance of the Far North Queensland Hospital Foundation, which holds an annual cycling charity challenge event. The details of the event are presented in the following section. The survey instrument was administered online via the Qualtrics web survey host. The survey opened on October 5, 2012, a few days after the completion of the event, and remained open until October 14, 2012. The survey collected information regarding participants' sociodemographic characteristics (gender, age, education, occupation, and income), their place of residence, and their involvement in cycling-related activities. A total of 213 usable responses were received by the survey cut-off date, a response rate of $42.6 \%$. A prize draw was offered to enhance response rates, and prizes included one free entry to the 2013 event or an AU\$300 gift voucher at a local cycling shop. Data collected were analyzed using the SPSS statistical software package.

Several research limitations may be noted. First, no measures of actual physical health and wellbeing were collected as part of the survey. Instead, the sociodemographic profiles of event participants are compared with the known profiles of lifestyle disease "at-risk" groups in Australia. Data on actual physical health will be collected as part of a separate study on the outcomes of charity challenge participation, whereas initial mental health outcomes of charity challenge participation have been investigated, with encouraging results (Coghlan \& Filo, 2013). The approach taken in this article reflects the exploratory and early stage nature of research into 
charity challenges more broadly, and the nascent relationship with the event organizers who provided access to their event participants. Current research into participant profiles is designed to establish a track record to encourage more detailed research into the role of charity challenge events as a form of lifestyle medicine. Second, the sample is limited to only one event, limiting the generalizability of the findings, while opening up new questions for further research. Finally, statistical comparisons were not possible as the study relied on a mix of primary and secondary data.

\section{The Yellow Pages Cardiac Challenge}

The Yellow Pages Cardiac Challenge is an annual cycling charity challenge that involves a 3-day tour of the local region, $335 \mathrm{~km}$ of sealed road from Cairns to Cooktown. It is a form of sport tourism according to Weed (2006), as it includes a travel element involving an overnight (3-day) tour of Far North Queensland and some form of 'sport' activity (i.e., cycling) as active participants. The challenge was first conducted in 2007, and 122 riders participated in the event. Each subsequent year has seen an increase of $20-40 \%$ in the number of participants signing up to complete the ride. The 3-day itinerary takes cyclists along part of Queensland's tourist route known as the Great Tropical Drive. It covers two mountain ranges as part of the challenge (Kuranda and Desailey ranges), traversing undulating savannah country for the majority of the ride. It also incorporates a number of regional towns where lunch stops and overnight campsites are organized for cyclists, volunteers, riders' support vehicles, and event organizers.

During the ride itself, cyclists travel in packs of 10-12 participants and each pack caters to different experience and fitness levels. Depending on the level of the pack, participants may spend up to 7 hours cycling each day, although the faster packs may only ride for 3-4 hours each day. The riders are accompanied by army support vehicles, volunteers to care for the riders, two first aid vehicles (one for the riders and one for their bicycles), and an entourage of supporters, friends, and relatives. Participants pay a registration fee of AU\$300, and must raise at least $\mathrm{AU} \$ 300$ for the charity recipient, the Far North Queensland Hospital Foundation. To date, nearly AU\$1,000,000 has been raised for the cardiac unit at the local Cairns hospital charity through this charity challenge.

\section{Results}

Results are presented in two major sections. First, the participants' sociodemographic characteristics are presented based upon the results of the survey. This is followed by a comparison of charity challenge participants' characteristics and the sociodemographic characteristics of "at-risk" groups for lifestyle-related diseases (Aim 1). Second, the cycling involvement and experience of charity challenge participants are reported (Aim 2). Comparative data (charity challenge participants and "at-risk" groups) are presented as tables for comparative purposes.

\section{Participant Sociodemographic \\ Characteristics and Comparative Results}

This section of the results describes the demographic attributes of respondents to the 2012 Yellow Pages Cardiac Challenge Participant survey. Age and gender distributions are first reported, followed by education, employment, and occupational characteristics, personal income, and respondents' usual state of residence. Wherever possible, comparisons are made with the most recent Australian population census based on 21,507,717 residents in Australia in 2011 (ABS, 2012), to gain a better comparative understanding of the profile of charity challenge event participants.

Of those who responded to this survey $(n=213)$, $64.4 \%$ were male, $35.6 \%$ were female, and $10.3 \%$ did not specify their gender. The dominant age category was 40-44 years (21.3\%), followed by 35-39 years and 50-54 years (both 17.5\%). Persons aged between 45 and 49 years accounted for $13.7 \%$ of respondents. Respondents under the age of 35 accounted for $16.9 \%$ of the total respondents and respondents over the age 55 accounted for $13.1 \%$. The mean age for the entire sample was 44 years (SD 11 years). The mean age of male respondents was 45.0 years (SD 12 years), while the mean age of female respondents was 41.5 years (SD 8.8 years). 
Table 2

Current Employment Status of Respondents $(n=209)$

\begin{tabular}{lr}
\hline Employment Status & $\%$ \\
\hline Employed full-time $^{\mathrm{a}}$ & 80.4 \\
Employed part-time & 7.9 \\
Retired & 6.9 \\
Full-time $^{\mathrm{a}}$ student & 3.2 \\
Unemployed, looking for full-time $^{\mathrm{a}}$ work & 1.1 \\
Unemployed, not looking for work & 0.5 \\
\hline
\end{tabular}

${ }^{a}$ Defined by the ABS as 35 or more hours per week.

The 2012 Yellow Pages Cardiac Challenge attracted a well-educated group of participants, with $50.5 \%$ of respondents possessing either a bachelor (34.5\%) or postgraduate degree (15.5\%). Another $21 \%$ of respondents reported completing secondary school, whereas $15 \%$ had a TAFE or trade certificate and $11 \%$ had a graduate diploma. The proportions of respondents with a bachelor degree or postgraduate degree far exceeded the Australian national proportion as determined in the 2011 Australian Population and Housing Census. Only $14.3 \%$ of Australians possessed (post)graduate qualifications, compared with $67 \%$ of this sample.

Those employed on a full-time basis constituted the vast majority of respondents. Table 2 highlights that $80.4 \%$ of respondents were employed on a full-time basis (35 or more hours each week). The remainder of respondents were employed parttime $(7.9 \%)$ or were retired (6.9\%), with only $1.1 \%$ unemployed and looking for full-time work and $3.2 \%$ in full-time studies.

Consistent with the large proportion of respondents that possess university qualifications, the majority of respondents were employed in professional fields (36.3\%). Managers and administrators were also quite prevalent (33.2\%), whereas only small numbers of respondents appeared in the remaining occupational categories such as tradespersons (5.3\%), clerical positions $(4.8 \%)$, or production and transport workers (1.1\%).

Again, consistent with the predominance of respondents engaged in professional and managerial occupations, respondents exhibited mostly high levels of weekly gross income. Figure 1 illustrates that a vast majority of respondents (31.6\%) earn in excess of AU\$1,800 per week before tax. This finding is not surprising given the statistics surrounding participants' education and employment, and is also consistent with income data found by other studies of cyclists in Australia (Lamont, 2009b). Persons who earn AU\$1,000 per week or more accounted for $76.6 \%$ of the sample. Persons earning AU\$600 per week or less accounted for $10.2 \%$ of respondents. Comparisons with the ABS (2012) census data of 2011 suggest that participants in the event generally earn higher than average incomes, as illustrated by the significantly higher proportions of respondents in the higher income categories, compared to the proportions for the Australian general population (median personal income: AU\$577).

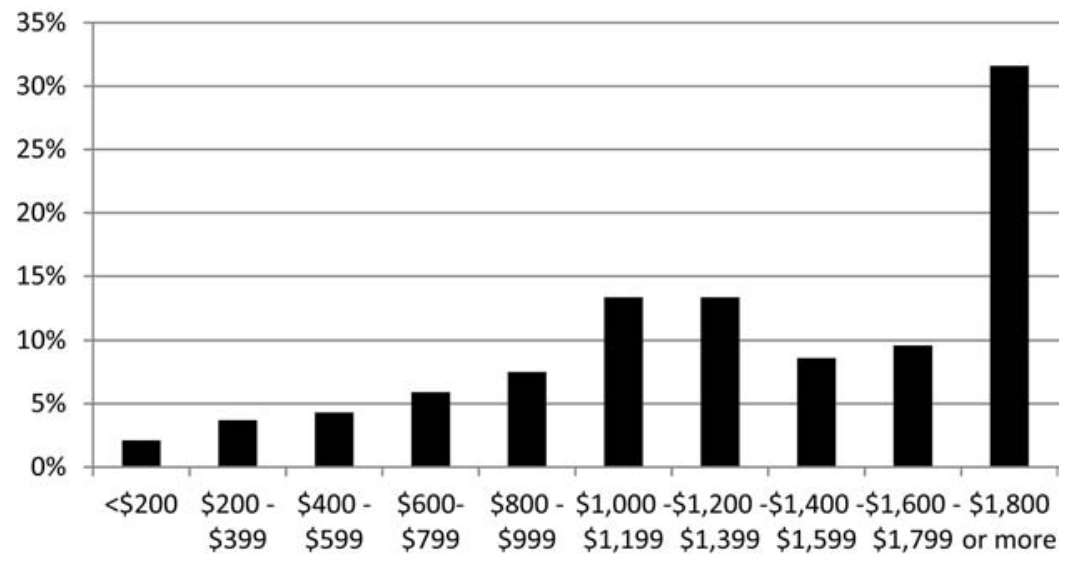

Figure 1. Personal weekly income before tax $(n=187)$. 
Finally, respondents were asked to provide their postcode to establish their usual place of residence. Respondents to this survey were mostly from the Cairns region (74.2\%), followed by North Queensland (8.0\%), other regions of Queensland (2.8\%), Australia (excluding Queensland) (3.8\%), and one international respondent from the UK. Participants in this case could be considered "regional and/or remote," although this result may reflect the community-based nature of the event and may not be generalizable to other event participants.

Comparing these results with the profiles of "atrisk” groups, we note some overlap. Table 3 summarizes these data and indicates some overlap in gender, age, occupation, and residency location, and level of activity between the two groups.

\section{Cycling Experience and Motivation to Participate}

Respondents were asked a series of questions regarding their level of cycling experience, their involvement with cycling, and their cycling-related motivations to participate in the 2012 Cardiac Challenge. Collecting these data enables us to ascertain if cycling charity challenge events appeal only to the "converted" (i.e., active cyclists who are already heavily involved in the sport), or whether these events provide a mechanism to engage new individuals in the sport of cycling. Of the 213 cyclists who responded to this question, $35.9 \%$ had participated in previous Cardiac Challenge events. Repeat participants had most often participated in one other event (52\% of repeat participants), and usually this was the 2011 Cardiac Challenge. Five respondents (8\% of repeat participants) had taken part in all six events. More generally, respondents had a variety of levels of experience as riders. Figure 2 indicates how many years respondents had been cycling prior to the 2012 Cardiac Challenge Event. Over 50\% of the respondents have been riding for 3 years or less, whereas $20 \%$ of respondents had been riding for more than 10 years. Respondents were also asked how many times they had participated in cycling events prior to the 2012 Cardiac Challenge. Again, over 50\% of respondents have been involved in cycling events for 3 years or less, and just over a third (35\%) had been involved in cycling for more than 6 years.

When asked how many recreational rides (i.e., less than 24 hours in duration, for exercise or pleasure) respondents undertake on average each week, $7.5 \%$ replied that they did not take part in any recreational rides. The majority of respondents (67.5\%) said that they cycle one to three times per week, and the remaining $25.2 \%$ cycle four times or more per week. In addition, respondents were asked a number of questions to determine their level of specialization and skill in cycling activities. Again, this information helps determine whether cycling charity challenges are "preaching to the converted" or whether novice riders are being encouraged to

Table 3

Sociodemographic Characteristics of cycling Charity Challenge Participants and “At-Risk” Groups

\begin{tabular}{|c|c|c|c|c|}
\hline & \multirow{2}{*}{$\begin{array}{l}\text { Participants in } \\
\text { Yellow Pages } \\
\text { Cardiac Challenge }\end{array}$} & \multicolumn{3}{|c|}{ “At-Risk” Groups of Lifestyle-Related Disease } \\
\hline & & Obesity $^{\mathrm{a}}$ & Cardiovascular Disease $^{\mathrm{b}}$ & Psychological Distress $^{\mathrm{C}}$ \\
\hline Gender & Male & Male & Male and females & Females \\
\hline Age & Middle to late adulthood & Middle to late adulthood & Middle to late adulthood & Young adults \\
\hline Education & Tertiary education levels & Year 11 completed & Data not available & Lower education levels \\
\hline Occupation & $\begin{array}{l}\text { F/T employment } \\
\text { managerial and } \\
\text { professionals }\end{array}$ & $\begin{array}{l}\text { F/T employment, } \\
\text { machinery operators, } \\
\text { managers }\end{array}$ & Data not available & $\begin{array}{l}\text { F/T employment } \\
\text { managerial and } \\
\text { professionals }\end{array}$ \\
\hline Income & High income & Lowest income & Lowest income & All income groups \\
\hline Location & Regional & Regional and remote & Regional and remote & Regional and remote \\
\hline Physical activity & Cycle 1-3 days/week & Exercise 2 days or less & Exercise 2 days or less & Exercise 2 days or less \\
\hline
\end{tabular}

Italic indicates possible overlaps between the groups.

aSource: ABS (2011).

'Source: AIWH (2011).

'Source: APS (2012a, 2012b). 


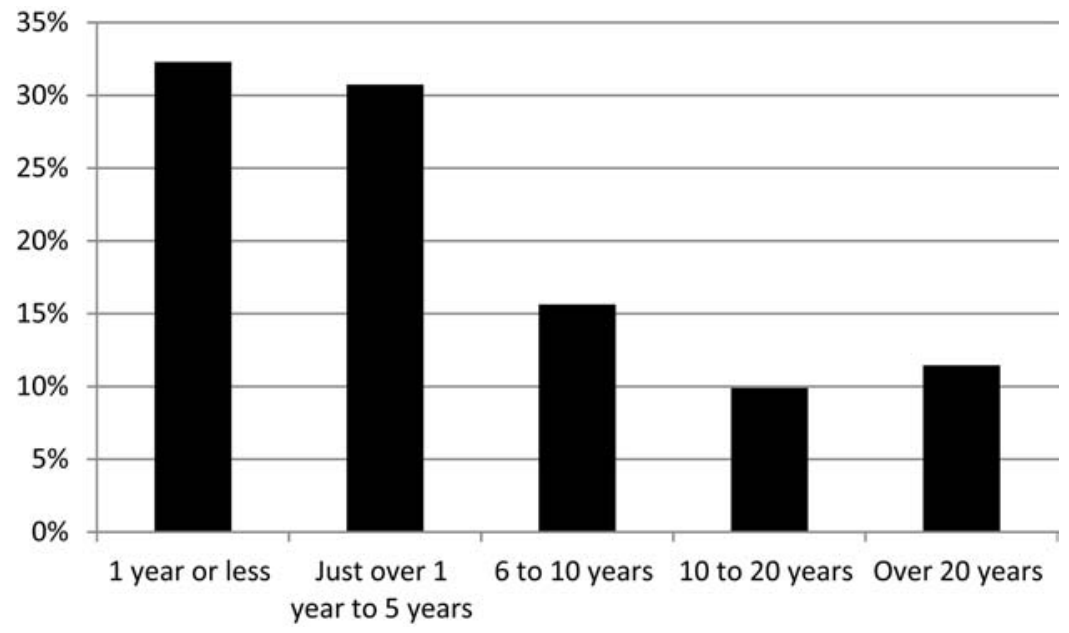

Figure 2. Distribution of responses relating to how many years participants had been cycling prior to the 2012 Cardiac Challenge $(n=192)$.

take up the activity. Respondents were asked how competent they felt in undertaking certain cyclingrelated tasks. Overall, the respondents appeared to feel relatively competent at all the tasks; $94.8 \%$ of respondents felt competent or very competent to ride in a straight line; $90.7 \%$ of respondents felt competent or very competent to safely ride in a pack of cyclists; $87.1 \%$ of respondents felt competent or very competent to ride defensively in traffic; $66.5 \%$ of respondents felt competent or very competent to undertake basic mechanical tasks; and $64.5 \%$ of respondents felt competent or very competent to take sharp corners at speed.

To explore charity challenge participants' level of involvement with cycling, respondents were asked to provide their level of agreement to a series of statements to determine how central cycling is in their daily lives. Results were recording on a 7-point scale, where 1 indicates strong disagreement and 7 indicates strong agreement. Table 4 shows the results of these questions and indicates the majority of respondents were only slightly in agreement with the different statements.

Finally, respondents were asked a series of questions regarding their cycling-related motivations for participating in the 2012 Cardiac Challenge. These covered a total of 38 items and respondents were asked to rate their level of agreement with each of the 38 statements on a 7-point scale, where 1 indicates strong disagreement and 7 indicates strong agreement. The most important cycling-related motivations for participating in the event are shown in Table 5. Interestingly, the opportunity to improve physical health was rated as the second most important motivation for respondents. Engaging in four (i.e., being active, relationships, achievement, and

Table 4

Statement About How Central Cycling Is to the Daily Lives of Participants $(n=200)$

\begin{tabular}{lcc}
\hline Statements About Cycling as a Central Part of Life & Mean & SD \\
\hline I enjoy discussing cycling with my friends & 5.28 & 1.104 \\
When I am cycling I can really be myself & 4.82 & 1.296 \\
You can tell a lot about a person when you go cycling with them & 4.47 & 1.260 \\
Cycling says a lot about who I am & 4.28 & 1.435 \\
I find a lot of my life is organized around cycling & 4.26 & 1.398 \\
Most of my friends are in some way connected to cycling & 3.93 & 1.545 \\
\hline
\end{tabular}


Table 5

Statement About How Central Cycling Is to the Daily Lives of Participants $(n=200)$

\begin{tabular}{lcr}
\hline Cycling-Related motivation & Mean & SD \\
\hline Cycling is an activity that you can do at any age & 6.33 & 0.731 \\
Cycling allows me to set new goals related to my health and fitness & 6.17 & 0.808 \\
Cycling at the event allows me to meet new people & 6.13 & 0.821 \\
Cycling gives me a strong sense of accomplishment & 6.13 & 0.899 \\
I enjoy the feeling of exhilaration after I have ridden my bike & 6.09 & 0.925 \\
Cycling allows me to exercise and enjoy pleasant surrounds at the same time & 6.05 & 0.861 \\
The event's location is a great place to cycle & 6.02 & 0.985 \\
\hline
\end{tabular}

positive emotions) of the five most common pathways to well-being (New Economics Foundation, 2011) were listed as four of the top five motivations to participate in the event.

\section{Discussion and Conclusions}

Notwithstanding the limitations of the data presented in this study, the results do highlight a number of interesting findings. First, it may be argued that there is some overlap in the sociodemographic profiles of charity challenge participants and lifestyle disease "at-risk" groups. This is particularly true of gender (albeit a broad category), age, place of residence, and, to some extent, levels of physical activity. Although the overlap between profiles is not a complete one, it is likely that the preevent training and actual event participation will have positive benefits on participants by encouraging them to become active in a new sport. Signing up to undertake a charity challenge may encourage new patterns of behavior that facilitate becoming more active and taking greater responsibility for their long-term health.

Such results would be in line with the process of internalization described by Deci and Ryan (2000) in their self-determination theory. They note that some forms of extrinsic motivational regulation can be internalized under certain conditions. The internalization process may occur through internalized regulation, whereby individuals recognize the benefits of undertaking the given activity, or integrated regulation, whereby individuals endorse the purpose and value of the behavior, and fully integrate it into their lifestyle. In this case, the behavior becomes more self-determined, and therefore sustained. Integrated regulation represents the optimal health outcome for charity challenges, and has been noted in other sports research (Lamont \& Kennelly, 2012), but has yet to be studied in a sport tourism context. Certainly, the respondents in this study could not be characterized as "serious" cyclists based upon the level of involvement questions (Table 3) and frequency of participation in recreational cycling or in cycling events; in general respondents did not see cycling as highly central to their lives, with the lowest scores attributed to the following statements: "Most of my friends are in some way connected to cycling" and "I find a lot of my life is organized around cycling." Moreover, the participants themselves nominated the motivation "cycling allows me to set new goals related to my health and fitness" as a key motivation for participating in the event. These results are encouraging as it suggests that charity challenge events are not "preaching to the converted" but are actually encouraging changes in behavior that may play an important role in the development of lifestyle medicine.

Interestingly, the evaluation of outcomes from mass participation events and health promotion events is generally unknown, and has been identified as a major research gap (Maddock, 2006; Murphy \& Bauman, 2007; Whitelaw \& Watson, 2005). We also know little about the differences between the profiles of participants in mass participation cycling events, competitive cycling events, cycle tourists, and cycling charity challenge participants, although it is likely that the former both attract participants with a history of active involvement in cycling (Bowles, Rissel, \& Bauman, 2006), while the latter may attract individuals who are newer to cycling. More research in this new and emerging type of charitable sport tourism event will contribute to our understanding of how these 
events recruit participants who may not have been highly active prior to signing up for the challenge and therefore may reap the greatest positive physical health benefits from participating in the event.

In terms of mental health benefits, the overlap here may be even greater, particularly with regard to occupation and income, and in this particular case, place of residence. In addition, respondents identified being active, building new relationships, having a sense of achievement, and positive emotions as four of the top five motivations to participate in the event. These all related to subconcepts within self-determination theory, including sense of competence, autonomy, and relatedness, which are recognized pathways to well-being (Deci \& Ryan, 1985). Further opportunities to use charity challenges as "well-being interventions" are being investigated by the author. Based on a review of positive psychology-based interventions, these types of events appear to offer a number of characteristics that foster increased well-being. These include the recognized pathways of well-being listed above (being active, developing relationships and a sense of achievement, experiencing positive emotions), as well doing something meaningful and being engaged, both of which may be unique to charity challenge events. Other characteristics may include goal setting, activation of signature strengths, gratification through fundraising and completing the challenge, and finally sharing the positive experience with others. Although further research is needed in this area, it appears that charity challenge events offer promising opportunities to foster a sense of well-being and mental health in their participants.

In conclusion, this study highlights several knowledge gaps and raises a number of questions with regard to the role of cycling charity challenge events in promoting positive physical and mental health outcomes through increased cycling participation. First, we have no knowledge of the size and scope of this sector, nor have existing studies identified the participation rates and profiles of cycling charity challenge event participants more broadly. In addition, the evaluation of physical activity campaigns, and the direct physical health outcomes of participating in cycling charity challenge events are also an area for further investigation. Finally, evaluating the health awareness outcomes through participating in the event and encouraging others to support the events' health-related cause could also be investigated as part of our understanding of the valuable links between fundraising activities, health issues, and charity challenge event activities.

These results contribute to existing health policy debates by exploring events that can support lifestyle medicine initiatives and encourage newcomers to cycling to be active, see cycling as a means to improve physical health, build relationships with others, develop a sense of accomplishment, and experience positive emotions. The likely benefits of cycling charity challenges include raising funds for charity, enduring involvement in cycling, health benefits from increased participation in cycling, opportunities to connect with the self and others by engaging in "serious leisure" (Stebbins, 2001), greater awareness of the health issues (for the participants and the participants' supporters), and a sense of personal empowerment as a result of completing the challenge and realizing fundraising goals.

Further research could usefully explore the role of charity challenges as a form of lifestyle medicine, by adopting Deci and Ryan's notion of integrated regulation, whereby participants endorse the values of the activity and adopt it in their everyday lifestyle. Encouraging this type of integrated regulation may have important implications for public health policy, and cycling charity challenges could be designed to improve participant physical health and well-being and reduce the burden of lifestylerelated diseases on society as a whole.

\section{References}

Australian Institute of Health and Welfare. (2011). Cardiovascular disease: Australian racts 2011 (Cardiovascular disease series, Cat. No. CVD 53). Canberra: Author.

Australian Institute of Health and Welfare. (2012). Cardiovascular health. Retrieved March 2013, from http:// www.aihw.gov.au/cardiovascular-health/

Australian Bureau of Statistics. (2011). Overweight and obesity in adults in Australia: A snapshot, 2007-08. Retrieved from http://www.abs.gov.au/ausstats/abs@. nsf/mf/4842.0.55.001/

Australian Bureau of Statistics. (2012). 2011 census quickstats. Retrieved March 5, 2013, from http://www.censusdata.abs. gov.au/census services/getproduct/census/2011/quickstat/0

Australian Psychological Society. (2012a). Stress and wellbeing in Australia in 2012: A state of the nation survey. Retrieved January 2013, from http://www.psychology.org. au/Assets/Files/Stress\%20and\%20wellbeing\%20in\%20 Australia\%202011\%20Report\%20(2)\%5B1\%5D.pdf 
Australian Psychological Society. (2012b). Equity in health and wellbeing: Why does regional, rural and remote Australia matter? Retrieved January 2013, from http:// www.psychology.org.au/Content.aspx?ID $=3960$

Bowles, H. R., Rissel, C., \& Bauman, A. (2006). Mass community cycling events: Who participates and is their behaviour influenced by participation? International Journal of Behavioural Nutrition and Physical Activity, 3, 39-45.

Coghlan, A., \& Filo, K. (2013). Using constant comparison method and qualitative data to understand participants' experiences at the nexus of tourism, sport and charity events. Tourism Management, 35, 122-131.

Cohen, A. (1985). The symbolic construction of community. London: Tavistock.

Deci, E., \& Ryan, R. (1985). Intrinsic motivation and selfdetermination in human behavior. New York: Plenum Press.

Deci, E., \& Ryan, R. (2000). The "what" and "why” or goal pursuits: Human needs and the self-determination of behavior. Psychological Inquiry, 11(4), 227-268.

Egger, G., \& Egger, S. (2012). Lifestyle medicine: The Australian experience. American Journal of Lifestyle Medicine, 6(1), 26-30.

Everyday Hero. (2012). About us. Retrieved December 23, 2012, from http://www.everydayhero.com.au/home/ about us

Frederickson, B. L. (2001). The role of positive emotions in positive psychology: The broaden-and-build theory of positive emotions. American Psychologist, 56(3), 218-226.

Gable, S., Reis, H., Impett, E., \& Asher, E. (2004). What do you do when things go right? The intrapersonal and interpersonal benefits of sharing positive events. Journal of Personality and Social Psychology, 87(2), 228-245.

Goodwin, H., McCombes, L., \& Eckardt, C. (2009). Advances in travel philanthropy: Raising money through the travel and tourism industry for charitable purposes. Retrieved from http://www.wtmwrtd.com/files/2_nov_ wtm tp final report.pdf

Haenfler, R., Johnson, B., \& Jones, E. (2012). Lifestyle movements: Exploring the intersection of lifestyle and social movements. Social Movement Studies, 11(1), 1-20.

Lamont, M. (2009a). Reinventing the wheel: A definitional discussion of bicycle tourism. Journal of Sport and Tourism, 14(1), 5-23.

Lamont, M. (2009b). Independent bicycle tourism in Australia: A whole tourism systems analysis. Unpublished Ph.D. thesis, School of Tourism \& Hospitality Management, Southern Cross University.

Lamont, M., \& Kennelly, M. (2012). A qualitative exploration of participant motives among committed amateur triathletes. Leisure Sciences, 34, 236-255.
Lyons, K., \& Wearing, S. (2008). All for a good cause? The blurred boundaries between volunteering and tourism. In K. Lyons \& S. Wearing (Eds.), Journeys of discovery in volunteer tourism (pp. 147-154). Wallingford, UK: CABI.

Lyubomirsky, S., Sheldon, K., \& Schkade, D. (2005). Pursuing happiness: The architecture of sustainable change. Review of General Psychology, 9(2), 111-131.

Massey, S., Cameron, A., Ouellette, S., \& Fine, M. (1998). Qualitative approaches to the study of thriving: What can be learned? Journal of Social Issues, 5(1), 337-355.

Maddock, J. (2006). Evaluation of community-based physical activity programs. Evaluation and Program Planning, 29, 240-241.

McIntyre, N. (1989). The personal meaning of participation: Enduring involvement. Journal of Leisure Research, 21, 167-179.

Murphy, N., \& Bauman, A. (2007), Mass sporting and physical activity events-Are they "bread and circuses" or public health interventions to increase population levels of physical activity? Journal of Physical Activity and Health, 4, 193-202.

New Economics Foundation. (2011). Sustainable development and well-being: Relationships, challenges and policy implications. London: The Department for Environment, Food and Rural Affairs.

Roubanis, J. L. (2008). Consumerism and voluntary simplicity lifestyle between US and Japanese female college students. Family and Consumer Sciences Research Journal, 37(2), 210-218.

Seligman, M. E. P., Parks, A. C., \& Steen, T. (2004). A balanced psychology and a full life. Philosophical Transactions of the Royal Society of London B, 359, 1379-1381.

Seligman, M. E. P., Steen, T. A., Park, N., \& Peterson, C. (2005). Positive psychology progress: Empirical validation of interventions. American Psychologist, 60(5), 410-421.

Stebbins, R. (2001). Serious leisure. Society, 28(4), 53-57.

Tourism Research Australia. (2013). National visitor survey. Retrieved from http://www.tra.gov.au/publications/ latest-nvs-report.html

Vallerand, R. (2007). Intrinsic and extrinsic motivation in sport and physical activity: A review and look at the future. In G. Tenenbaum \& R. C. Eklund (Eds.), Handbook of sport psychology (3rd ed., pp. 59-83). New York: John Wiley.

Weed, M. (2006): Sports tourism research 2000-2004: A systematic review of knowledge and a meta-evaluation of methods. Journal of Sport \& Tourism, 11(1), 5-30.

Whitelaw, S., \& Watson, J. (2005). Whither health promotion events? A judicial approach to evidence. Health Education Research, 20(2), 214-225. 